\title{
Feeling Is Believing: A Secure Template Exchange Protocol
}

\author{
Ileana Buhan, Jeroen Doumen, Pieter Hartel, and Raymond Veldhuis \\ University of Twente, Enschede, The Netherlands \\ \{ileana.buhan, jeroen. doumen, pieter.hartel, \\ r.n.j.veldhuis\}autwente.nl
}

\begin{abstract}
We use grip pattern based biometrics as a secure side channel to achieve pre-authentication in a protocol that sets up a secure channel between two hand held devices. The protocol efficiently calculates a shared secret key from biometric data. The protocol is used in an application where grip pattern based biometrics is used to control access to police hand guns.
\end{abstract}

Keywords: Ad-hoc authentication, fuzzy cryptography, biometrics.

\section{Introduction}

We are developing smart guns with grip pattern biometrics [11] for the police, to reduce the risk of officers being shot with their own weapon in case of a take away situation [7]. In this scenario a police handgun authenticates the owner by grip pattern biometrics integrated with the grip of the gun. Police officers often work in teams of two and each officer must be able to fire the other officer's weapon. Normally, teams are scheduled in advance so that appropriate templates can be loaded into the weapons at the police station. However, in emergency situation this is not possible; in this case police officers have to team up unprepared and exchange templates in the field. Biometric data is sensitive information thus during the exchange the templates must be protected. Officers may work with colleagues from other departments, even from neighboring countries, so a shared key, or a public key infrastructure where the certificate associated with these keys must be verifiable on-site is not realistic. Also, one cannot expect a police officer to perform some complicated interfacing operation with his gun in the field.

In this paper we present a solution that is both simple and effective. Each police officer owns a gun, that holds the owners stored biometric grip pattern template and is equipped with a grip pattern sensor and a short range radio. The users swap the devices, so that each device can measure the grip pattern of the other user. The devices are then returned to their owners. Each device now contains a genuine template of its owner and a measurement of the other user, called guest. The devices calculate a common key from the owner's template and the guest measurement. The act of the guest putting her hand on the user's device corresponds to sending a message on a secure side channel. Therefore, we have termed our protocol Feeling is Believing (FiB).

Securing the exchange of biometric templates is an instance of the pairing problem. As described by Saxena [9] the pairing problem is to enable two devices, which share no prior context, to agree upon a security association that they can use to protect their 
subsequent communication. Secure pairing must be resistant to a man-in-the-middle adversary who tries to impersonate one or both of these devices in the process. To achieve secure pairing one cannot rely on any previously shared secret information. This problem can be solved in cryptography by the issue of certificates. As said before, due to the ad-hoc nature of the pairings we cannot rely on an existing communications channel to a certification authority that could validate certificates. Our approach is to use an additional physically authenticated side channel which is governed by humans.

Related Work. Balfanz et al. [9] propose physical contact between devices. This type of channel has the property that the user can control precisely which devices are communicating. The authors extend this approach to location limited channels where they propose short range wireless infrared communication where the secret information is communicated when devices are in the line of sight. McCune et al. [6] propose to use a visual channel and make photographs of the hash codes of the public keys. This represents a major breakthrough especially from the point of user friendliness. In the same line of work, Googrich et al. [4] propose a human assisted authentication audio channel as a secure side channel. They use a text to speech engine for vocalizing a sentence derived from the hash of a device's public key. The pre-authentication channel is used mostly to authenticate public-keys. The hash of the public key is either vocalized [4] or photographed [6]. Others [12[10], use a Diffie-Hellman like key agreement scheme where short sequences transmitted on the private channel authenticate the key sent on the main channel. Our protocol is a Diffie-Hellman like protocol in the sense that both parties equally contribute to the session key. FiB achieves mutual authentication and the partial keys are extracted from the biometric identification data of the individual.

Contribution. FiB can perform secure biometric template exchange in an ad-hoc situation, its main merit being user friendliness. We stress that the application domain for $\mathrm{FiB}$ is more general than the gun application. FiB can securely exchange template for any type of biometric system. FiB is formally verified to prove the security and origin authentication of templates. The workload of an intruder who is trying to guess the session key is evaluated in 9 different scenarios. To the best of our knowledge, this is the first time that a biometric is used as a location limited channel. Using biometrics and cryptography together brings up challenges. Biometric data is usually noisy. However the noise is not reproducible or uniformly random thus cryptography algorithms have difficulties in adjusting to this noise. We propose a new method for correcting errors in the keys generated from biometric data using a user specific error profile.

\section{FiB Protocol Description}

FiB solves the problem of secure template exchange and provide a solution for securing the ad-hoc transfer of private information between mobile, biometrically enabled devices in a scenario where no pre-distributed keys are available. The main threat is an intruder loading his template into one of the participating devices. Thus, we require the authorization of the device owner before his template is transferred. To preserve the privacy of the biometric template, it must be encrypted before being sent out. 
Fuzzy Key Extraction. Before we turn to the protocol, we introduce the main tool. A fuzzy extractor as defined by Dodis et al. [1] is a public function that extracts robustly a binary sequence $K$ from a noisy measurement $F$ with the help of some public string $H$. Enrollment is performed by a function Gen, which on the input of the noise free biometric $T$ and the chosen binary string $K$ will compute a public string $H=\operatorname{Gen}(T, K)$. During authentication, a second function Reg takes as input a noisy measurement $F$ and the public string $H$ and outputs a binary string $K^{\prime}=\operatorname{Reg}(F, H)$. In a perfect world, $K^{\prime}=K$ but in reality they might be slightly different. However, we expect that keys are close in terms of their Hamming distance when $T$ and $F$ are similar enough. Both $T$ and $F$ are multidimensional feature vectors and while for most of the features the authentication will work correctly, for some feature the noise might be too large. We write $K^{\prime}=K+e$, where we assume that the Hamming weight of the error $e$, denoted with wt $(e)$ satisfies wt $(e) \leq t, 0 \leq t \ll n$.

FiB Protocol preliminaries. Before we delve into the description of the protocol, which represents the core of our solution, we describe the context. Enrollment of users takes place before the protocol starts. During enrollment a low noise measurement $T$ is taken, for each user. A key $K$ of length $n$ is generated and function Gen(T,K) outputs the helper data $H$. Then the user specific error profiles $E$ is computed. The error profile is used by the protocol to lower the error rates of the biometric system, see subsection 4 This information is loaded into the device of the user. After the enrollment we have achieved that: (1) the identity of the user can be verified by his own device, and (2) a device is prepared to run the FiB protocol which allows secure template transfer so that another device can verify the identity of the user.

The Protocol. The principals that interact during the protocol are two devices $D_{a}$ and $D_{b}$. Before the protocol starts each device knows the data of its owner, i.e. the template, the helper data constructed from the template, the key and the error profile. Hence initially $D_{a}$ knows $\left\{T_{a}, H_{a}, K_{a}, E_{a}\right\}$ and $D_{b}$ knows $\left\{T_{b}, H_{b}, K_{b}, E_{b}\right\}$. The message flow of the FiB protocol is shown in Figure 1. By $K_{a}^{\prime} \| K_{b}^{\prime}$ we mean a combination of $K_{a}^{\prime}$ and $K_{b}^{\prime}$, for example concatenation.

Actions of device $D_{a}$ (Initiator). We assume that $D_{a}$ starts the protocol. When an unknown grip pattern is detected, $D_{a}$ broadcasts its helper data $H_{a}$ on the wireless channel. $H_{a}$ is constructed such that it does not reveal any significant information about $T$ or $K$. Upon receiving message 4 from $D_{b}, D_{a}$ uses the received $H_{b}$ and $F_{b}$ to extract $K_{b}^{\prime}$. The second part of the message is used to help $D_{a}$ recover $K_{a}^{\prime}$. Since $K_{a}$ and $K_{a}^{\prime}$ are close, $D_{a}$ can use the error profile $E_{a}$ to recover $K_{a}^{\prime}$ by flipping carefully chosen bits in $K_{a}$ until it can successfully decrypt $\left\{F_{a}\right\}_{K_{a}^{\prime}}$. Since $D_{a}$ can recognize a measurement coming from its own user, $D_{a}$ can check the decryption results. When $D_{a}$ successfully finds $K_{a}^{\prime}$ it sends message 7 to $D_{b}$. $D_{a}$ verifies in step 11 that $T_{b}$ matches the measurement received on the secure side channel in step 1.

Actions of device $D_{b}$ (Responder). Device $D_{b}$ receives $H_{a}$ and detects an unknown grip pattern, $F_{a}$. The rest of the operations are similar to those of $D_{a}$. Both participants have to perform the same amount of computation.

$\mathrm{FiB}$ is a solution that offers confidentiality during data transfer and authentication. However, FiB does not guarantee what happens to the templates after the protocol ends. 


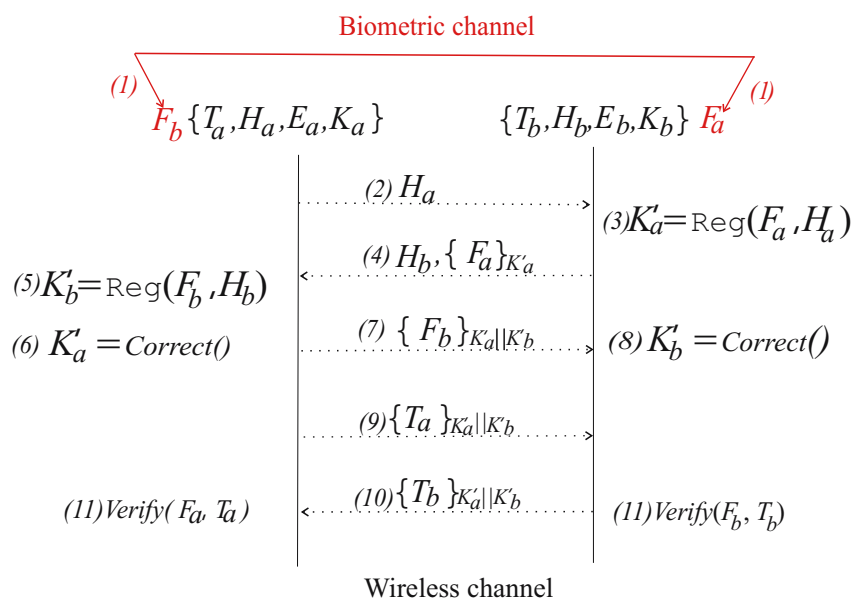

Fig. 1. FiB protocol

We emphasize that loading a template into ones device is similar to handing over a key to the device. At any time the owner of the template can access that device. In the scenario of the smartgun we assume that the sensitive information is stored in a tamper resistant storage environment where the templates cannot be "taken out".

\section{Security Evaluation for FiB Protocol}

There are two distinct, rigorous views of cryptography that have been developed over the years. One is a formal approach where cryptographic operations are seen as black box functions represented by symbolic expressions and their security properties are modelled formally. The other is based on a detailed computational model where cryptographic operations are seen as strings of bits and their security properties are defined in terms of probability and computational complexity of successful attacks. In the following we look at both aspects of security.

\subsection{Formal Verification of the FiB Protocol with CoProVe}

We have formally verified that FiB satisfies secrecy of the templates and mutual authentication. The adversary, named Eve, is a Dolev-Yao [3] intruder that has complete control of the communication channel. She can listen to, or modify messages on the main communication channel between the devices but cannot access the secure side channel. The tool used for this purpose is the constraint based security protocol verifier CoProVe by Corin and Etalle [2]. An earlier version of the protocol was verified and found buggy, the published version of the protocol above fixes the flaw found. A (security) protocol is normally verified using a model of the protocol, to avoid getting bogged down in irrelevant detail. The quality of the model then determines the accuracy of the verification results. The basic difference between a protocol and a model lies in the assumptions made when modelling the protocol. We believe that the following assumptions are realistic: 
1. No biometric errors. We assume that the correction mechanism always works perfectly and thus the initiator knows the key used by the sender. Thus, we look only at complete protocol rounds. When the initiator cannot work out the key the protocol is aborted. In this case we assume that Eve cannot work out the key either.

2. Modelling the secure side channel. We assume that when the protocol starts device $D_{a}$ knows $F_{a}$ and device $D_{b}$ knows $F_{b}$ while Eve knows neither because she cannot eavesdrop on the secure side channel.

3. Classifier based verification in step 11 removed. Because systems without an equational theory such as CoProVe cannot compare two terms, the last check before accepting a template cannot be modelled. This check prevents an intruder modifying messages 9 and 10 in the protocol.

We have verified the model in figure 1 with the assumptions above. We argue that the above abstractions do not affect the secrecy and the authentication property. Verification with CoProVe explores a scenario in which one of the parties involved in the protocol plays the role of the initiator (i.e. the party staring the protocol) and the other plays the role of the responder. A third party, the intruder learns all message exchanged by the initiator and the responder. The intruder can devise new messages and send them to honest participants as well as replay or delete messages. Should the intruder learn a secret key and a message encrypted with that key, then the intruder also knows the message. This is the classical Dolev-Yao intruder [3].

We have explored two scenarios that we believe to be realistic and representative for real attacks. In the first scenario two honest participants Alice and Bob are plagued by a powerful intruder who is assumed to know $T_{b}$ (because the intruder might have communicated with Bob in a previous session).

In the scenario the intruder tries to load her own template $T_{i}$ into $D_{a}$ so as to be able to fire weapon $T_{a}$. This impersonation attack is found to be impossible by CoProVe under the assumption that the intruder does not know $F_{a}$ or $T_{a}$. This is a realistic assumption since these are not transmitted in clear and the key used to encrypt is computed from data sent over the secure side channel. Verification thus shows that the intruder cannot impersonate $D_{b}$ even though the intruder has knowledge of $T_{b}$.

In the second scenario one participant, Alice, has to deal with the intruder, who does not have useful initial knowledge. This scenario represents the case that Alice's device was stolen and the intruder tries to load his template into Alice's device and use it. Verification shows that $T_{a}$ remains secret when Alice is the initiator of the protocol. This means that an intruder cannot trick Alice into disclosing her template data if she does not provide the intruder a sample of her biometric data. When Alice is the responder and the intruder has full access to the device (i.e. the intruder can submit his own biometric data), $T_{a}$ will not be disclosed. This is because before the device sends anything useful on the wireless link the device will check whether his owner is there after step 3.

As a result in both scenarios we have verified the security and authentication authentication of responder to the initiator and authentication of initiator to responder.

\subsection{Intruder's Computational Effort of Guessing $K_{a}^{\prime} \| K_{b}^{\prime}$.}

To derive keys from fuzzy data we use a semi-known plain text attack in steps 6 and 8 , to recover the session key. This approach can raise a couple of natural questions: "If 
both Alice (device $D_{a}$ ) and Bob (device $D_{b}$ ) have to guess the session key, how much more difficult is for Eve (the intruder) to do the same?", "What happens if Eve already knows the templates of Alice and or Bob?", "What kind of guarantees is this protocol offering?" To answer these questions we study the following scenarios:

AE(0) No previous contact between Alice and Eve.

AE(1) Eve records a measurement of Alice's biometric.From the helper data sent out in the clear Eve constructs $K_{a}^{\prime \prime}$.

AE(2) Eve had a protocol run with Alice and knows $T_{a}$ and Eve can construct $K_{a}$.

We denote by $W(x \rightarrow y)$ the average number of trials that Eve, has to do to guess $y$ when she knows $x$ using the best guessing strategy. We analyze Eve's workload to guess $K_{a}^{\prime}$ in the three scenarios above.

In scenario $A E(2)$, Eve knows $K_{a}$ and has to guess $K_{a}^{\prime}=K_{a}+e$ where wt $(e) \leq t$. Since Eve has no information about the noise distribution, she has to correct up to $t$ errors. As the key length is $n$, there are $\left(\begin{array}{c}n \\ i\end{array}\right)$ different error patterns if the actual number of errors is $i$, thus on average she will have to guess:

$$
W\left(K_{a} \rightarrow K_{a}^{\prime}\right) \approx \frac{1}{2} \sum_{i=0}^{t}\left(\begin{array}{l}
n \\
i
\end{array}\right)+\frac{1}{2} .
$$

In scenario $A E(1)$, Eve knows $K_{a}^{\prime \prime}$ and has to guess $K_{a}^{\prime}$ where $K_{a}^{\prime \prime}=K_{a}+e^{\prime}$, thus $K_{a}^{\prime \prime}=K_{a}^{\prime}+e^{\prime}+e$. Since wt $\left(e^{\prime}+e\right) \leq 2 t$, Eve has workload:

$$
W\left(K_{a}^{\prime \prime} \rightarrow K_{a}^{\prime}\right) \approx \frac{1}{2} \sum_{i=0}^{2 t}\left(\begin{array}{l}
n \\
i
\end{array}\right)+\frac{1}{2} .
$$

In scenario $A E(0)$ Eve has no information on Alice thus she has to brute force all possibilities. Thus the number of trials is approximately:

$$
W\left(0 \rightarrow K_{a}^{\prime}\right) \approx \frac{2^{n}+1}{2} .
$$

Scenarios for Bob are analogous:

BE(0) No previous contact between Bob and Eve.

BE(1) Eve records a measurement of Bob.

BE(2) Eve had a previous round with Bob thus knows $T_{b}$ and can construct $K_{b}$.

Eve's workload for guessing $K_{b}^{\prime}$ is equal to guessing $K_{a}^{\prime}$ in the analogous scenario. To achieve her goal of loading her template in one of the devices, Eve has to guess $K_{a}^{\prime} \| K_{b}^{\prime}$ in all scenarios. Table 1 summarizes her workload. In each row we have the information that Eve knows about Bob and in the column the information that Eve knows about Alice. Due to the message flow in the protocol (see figure 1), Eve might have an advantage if she has information about Alice. Eve can intercept message (4), $\left\{F_{a}\right\}_{K_{a}^{\prime}}$ and recover $K_{a}^{\prime}$ if the biometrics allows for taking a decision on whether two measurements come from the same individual. This explains the plus sign between the work of guessing $K_{a}^{\prime}$ and the work of guessing $K_{b}^{\prime}$ in the columns where Eve has some 
Table 1. Guesswork required for Eve to compute the session key

\begin{tabular}{l|lll} 
& \multicolumn{1}{|c}{$\mathrm{AE}(0)$} & \multicolumn{1}{c}{$\mathrm{AE}(1)$} & $\mathrm{AE}(2)$ \\
\hline $\mathrm{BE}(0)$ & $W\left(0 \rightarrow K_{a}^{\prime}\right) \cdot W\left(0 \rightarrow K_{b}^{\prime}\right)$ & $W\left(K_{a}^{\prime \prime} \rightarrow K_{a}^{\prime}\right)+W\left(0 \rightarrow K_{b}^{\prime}\right)$ & $W\left(K_{a} \rightarrow K_{a}^{\prime}\right)+W\left(0 \rightarrow K_{b}^{\prime}\right)$ \\
$\mathrm{BE}(1)$ & $W\left(0 \rightarrow K_{a}^{\prime}\right) \cdot W\left(K_{b}^{\prime \prime} \rightarrow K_{b}^{\prime}\right)$ & $W\left(K_{a}^{\prime \prime} \rightarrow K_{a}^{\prime}\right)+W\left(K_{b}^{\prime \prime} \rightarrow K_{b}^{\prime}\right)$ & $W\left(K_{a} \rightarrow K_{a}^{\prime}\right)+W\left(K_{b}^{\prime \prime} \rightarrow K_{b}^{\prime}\right)$ \\
$\mathrm{BE}(2)$ & $W\left(0 \rightarrow K_{a}^{\prime}\right) \cdot W\left(K_{b} \rightarrow K_{b}^{\prime}\right)$ & $W\left(K_{a}^{\prime \prime} \rightarrow K_{a}^{\prime}\right)+W\left(K_{b} \rightarrow K_{b}^{\prime}\right)$ & $W\left(K_{a} \rightarrow K_{a}^{\prime}\right)+W\left(K_{b} \rightarrow K_{b}^{\prime}\right)$
\end{tabular}

knowledge about Alice. We can estimate an upper bound for the work load of Alice and Bob according to Pliam's [8] equation:

$$
W_{\text {Alice }}\left(K_{a} \rightarrow K_{a}^{\prime}\right) \leq \frac{1}{2}\left(\sum_{i=0}^{t}\left(\begin{array}{l}
n \\
i
\end{array}\right)+1\right)-\frac{1}{2} \sum_{i=0}^{t}\left(\begin{array}{l}
n \\
t
\end{array}\right) \cdot \sum_{j=1}^{n}\left|E_{j}(\sigma, q)-\frac{1}{2^{n}}\right|
$$

Here $E_{j}(\sigma, q)$ represents the distribution of the noise, which is described below. The best case scenario for Eve, however unlikely, is $[\mathrm{BE}(2), \mathrm{AE}(2)]$ when she had a previous round with both Alice and Bob. Even though she has both $K_{a}$ and $K_{b}$ her workload is at least twice as high compared to Alice or Bob. Moreover while for Eve all error patterns are equally likely, Alice and Bob have the error profile that makes recovery faster.

\section{Experimental Validation with Real Life Data}

The Correct function used during the FiB protocol uses a semi-known plain text attack to recover the key. We link the keys used in the protocol to the biometric data of a user using the template protection scheme proposed by Linnartz and Tuyls [5]. The purpose of using this function is to lower the error rate of the system by flipping bits on the result of function Reg in the order defined by the user specific user profile $E$.

Key search algorithm. In classical symmetric cryptography to decrypt a message encrypted with a key $K$ one must posses $K$. In particular, with a key $K^{\prime}$ that differs only in one bit from $K$, decryption will fail. The FiB protocol uses this apparent disadvantage of symmetric key cryptography as an advantage: $K^{\prime}$ is used to form the session key. The noise of the measurements is used as random salt [13] for the session key. The key search algorithm makes it possible to recover $K^{\prime}$. We start the key search by assuming there are no errors in $K^{\prime}$, and we use $K^{\prime}$ for decryption. If decryption fails we assume that we have a one bit error. We start flipping one bit of the key according to the position indicated by the error profile, until we have exhausted the error profile. Then we assume that two bits are wrong and we try all combinations of two bits from the error profile. Finally if we reach the limit on the number of trials we assume that the key is coming from an intruder. The recovery of $K^{\prime}$ is a semi-known plain text attack. When the correct value of $K^{\prime}$ is discovered the initiator will recognize the message encrypted with $K^{\prime}$. This is possible since the encrypted message is a biometric template. The initiator of the protocol possesses a fresh measurement of this template and hence is able to recognize a correct match. The verification is performed by a classifier based matching algorithm designed for this particular biometrics. 
Error profile computation. Linnartz et al. [5] propose a multiple quantization level system with odd-even bands. The embedding of binary data is done by shifting the template distribution to the center of the closest even-odd $q$ interval if the value of the key bit is a 1 , or to the center of an odd-even $q$ interval if the value of the key bit is a 0 . The calculation $\operatorname{Gen}\left(t_{i}, k_{i}\right)=h_{i}$ proceeds component wise as follows, where $i=1, n$ :

$$
\operatorname{Gen}\left(t_{i}, k_{i}\right)=h_{i}= \begin{cases}\left(2 p+\frac{1}{2}\right) \cdot q-t_{i}, & k_{i}=1 \\ \left(2 p-\frac{1}{2}\right) \cdot q-t_{i}, & k_{i}=0 .\end{cases}
$$

where $p \in \mathbb{Z}$ is chosen such that $h_{i} \leq 2 q$. During authentication the key is recovered by computing:

$$
\operatorname{Reg}\left(f_{i}, h_{i}\right)= \begin{cases}1, & 2 p q \leq f_{i}+h_{i}<(2 p+1) q \\ 0, & (2 p-1) q \leq f_{i}+h_{i}<2 p q .\end{cases}
$$

However, during Reg whenever the difference between the measured $f_{i}$ value and the template $t_{i}$ is greater than $\frac{q}{2}$ we get an error in the key computation. Each key bit is extracted independently. Thus, the error profile is a vector of length $n$. The $i$-th value of the error profile represents the probability of the $i$-th key bit to be computed wrongly. We assume that the features are independent and normally distributed. The error profile is computed component-wise, using the function:

$$
E_{i}(\sigma, q)=\sigma 2 \sqrt{2} \sum_{i=0}^{\infty} \int_{\frac{(1+4 i)}{2 \sqrt{2}} \frac{q}{\sigma}}^{\frac{(3+4 i)}{\sigma \sqrt{2}} \frac{q}{\sigma}} e^{-x^{2}} d x \approx \sigma 2 \sqrt{2} \int_{\frac{q}{\sigma 2 \sqrt{2}}}^{\frac{3 q}{\sigma 2 \sqrt{2}}} e^{-x^{2}} d x,
$$

During enrollment several different measurement have to be made for each user. The error profile is based on the fact that in practical situations the estimated standard deviation is different for different user. To compute the error profile we use the same data after dimensionality reduction that the classifier based matcher use.

Results. We implemented the key extraction described above. We wanted to see the influence of the correction mechanism on the overall error rates. The evaluation is performed on real life grip pattern biometric data collected from 41 police officers. A detailed description of this biometric can be found in Veldhuis et al. [11]. Each of the 41 officers contributed 25 different measurements. Approximately $75 \%$ of these samples(18), are used for training the algorithm and 25\% (7) are used for testing. First, we reduce the dimensionality of the data to 40 independent features. For training and testing we use the same data that is used for verification by the classifier based recognition algorithm.Second, we embed the key bits using Linnartz and Tuyls scheme above. Figures 2 presents the results obtained from the collected data. We offer three conclusions from this evaluation. The first conclusion is, as expected, the larger the quantization step the lower the FRR but the higher the FAR. We tested 8 different values for $q$, ranging from 1 to 5 in increments of 0.5 . We did not try larger values for $q$ because the FAR becomes unacceptably large. The second conclusion is that the influence of the correction algorithm is significant. For example for $q=3$, without correction the $\mathrm{FRR}=13.93 \%$ and the FAR=0\%. When we correct 1 bit the FRR goes down to $5.92 \%$, 


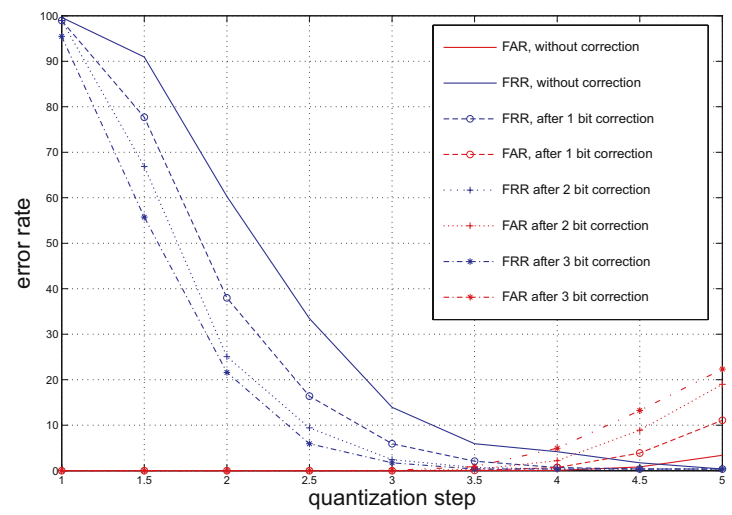

Fig. 2. Results on grip pattern data

while the FAR retains the same value $0 \%$. After correcting 2 bits the FRR goes down to $2.43 \%$ while the FAR remains equal to $0 \%$. Correcting 3 bits further reduces the FRR to $1.74 \%$ while the FAR increases only slightly to $0.07 \%$. The third conclusion is that the correction mechanism is stable, meaning that the effect of correction is independent of the time when data in collected. Data was collected during two sessions and the performance of the correction algorithm is similar on both sets.

\section{Conclusions}

The contributions of this paper are threefold. Firstly, we propose FiB a protocol that can exchange biometric templates securely even though no prior security association exists between the participants. We are confident in the guarantees offered by FiB because we formally verify the protocol to prove security and origin authentication. Also, from the information theoretic point of view the workload of Eve is at least double of that of Alice or Bob in the unlikely scenario where Eve has interacted with Alice and Bob and where Eve possess the same key material. Moreover, in this case the biometric has to be favorable to Eve in that she has to be able to verify wheatear two noisy measurements are coming from the same user or not. Secondly, for the first time we propose to use biometrics as a secure side channel. The advantage of using biometrics compared to any other types of side channels is the extreme user friendliness. Thirdly, a new correction mechanism for correcting biometric errors based on user specific error profile is proposed. We present an evaluation of the performance in terms of FAR and FRR and we show that the correction algorithm significantly improves the overall results. Our experiments show that by correcting only 1 bit in the overall key the FRR is reduced by approximately $50 \%$ while the FAR is not increased significantly. We believe that our approach can be applied to other types of biometrics.

\section{Acknowledgements}

The authors would like to thank Ricardo Corin and Sandro Etalle for helping formally verify the protocol using CoProVe. 


\section{References}

1. Boyen, X., Dodis, Y., Katz, J., Ostrovsky, R., Smith, A.: Secure remote authentication using biometric data. In: Cramer, R.J.F. (ed.) EUROCRYPT 2005. LNCS, vol. 3494, pp. 147-163. Springer, Heidelberg (2005)

2. Corin, R., Etalle, S.: An improved constraint-based system for the verification of security protocols. In: Hermenegildo, M.V., Puebla, G. (eds.) SAS 2002. LNCS, vol. 2477, pp. 326341. Springer, Heidelberg (2002)

3. Dolev, D., Yao, A.: On the security of public key protocols. Information Theory, IEEE Transactions on 29, 198-208 (1983)

4. Goodrich, M.T., Sirivianos, M., Solis, J., Tsudik, G., Uzun, E.: Loud and clear: Humanverifiable authentication based on audio. In: 26th IEEE International Conference on Distributed Computing Systems (ICDCS 2006), Lisboa, Portugal, 4-7 July 2006, p. 10. IEEE Computer Society Press, Los Alamitos (2006)

5. Linnartz, J.P, Tuyls, P.: New shielding functions to enhance privacy and prevent misuse of biometric templates. In: Kittler, J., Nixon, M.S. (eds.) AVBPA 2003. LNCS, vol. 2688, pp. 393-402. Springer, Heidelberg (2003)

6. McCune, J., Perrig, A., Reiter, M.: Seeing-is-believing: using camera phones for humanverifiable authentication. In: Security and Privacy, 2005 IEEE Symposium on, pp. 110-124. IEEE Computer Society Press, Los Alamitos (2005)

7. NJIT: Personalized weapons technology project, progress report. Technical report, New Jersey Institute of Technology (April 2001)

8. Pliam, J.O.: Guesswork and variation distance as measures of cipher security. In: Heys, H.M., Adams, C.M. (eds.) SAC 1999. LNCS, vol. 1758. Springer, Heidelberg (2000)

9. Saxena, N., Ekberg, J., Kostiainen, K., Asokan, N.: Secure device pairing based on a visual channel (short paper). SP, 306-313 (2006)

10. Vaudenay, S.: Secure communications over insecure channels based on short authenticated strings. In: Shoup, V. (ed.) CRYPTO 2005. LNCS, vol. 3621, pp. 309-326. Springer, Heidelberg (2005)

11. Veldhuis, R.N.J., Bazen, A.M., Kauffman, J.A., Hartel, P.H.: Biometric verification based on grip-pattern recognition. In: Security, Steganography, and Watermarking of Multimedia Contents VI, Proceedings of SPIE, San Jose, California, USA, January 18-22, 2004, vol. 5306, pp. 634-641 (2004)

12. Wong, F.L., Stajano, F.: Multi-channel protocols for group key agreement in arbitrary topologies. In: 4th IEEE Conference on Pervasive Computing and Communications Workshops (PerCom 2006 Workshops), Pisa, Italy, 13-17 March 2006, pp. 246-250. IEEE Computer Society Press, Los Alamitos (2006)

13. Wu, T.D.: The secure remote password protocol. In: Proceedings of the Network and Distributed System Security Symposium, NDSS 1998, San Diego, California, USA. The Internet Society (1998) 